\title{
Mapping of the local environmental changes in proteins by cysteine scanning
}

\author{
Yoichi Yamazaki $^{1,2}$, Tomoko Nagata ${ }^{1}$, Akihisa Terakita ${ }^{3}$, Hideki Kandori ${ }^{4}$, Yoshinori Shichida ${ }^{1}$ and \\ Yasushi Imamoto ${ }^{1}$ \\ ${ }^{1}$ Department of Biophysics, Graduate School of Science, Kyoto University, Kyoto 606-8502, Japan \\ ${ }^{2}$ Graduate School of Materials Science, Nara Institute of Science and Technology, Nara 630-0192, Japan \\ ${ }^{3}$ Department of Biology and Geosciences, Graduate School of Science, Osaka City University, Osaka 558-8585, Japan \\ ${ }^{4}$ Department of Materials Science and Engineering, Nagoya Institute of Technology, Nagoya 466-8555, Japan
}

Received October 10, 2013; accepted December 18, 2013

Protein conformational changes, which regulate the activity of proteins, are induced by the alternation of intramolecular interactions. Therefore, the detection of the local environmental changes around the key amino acid residues is essential to understand the activation mechanisms of functional proteins. Here we developed the methods to scan the local environmental changes using the vibrational band of cysteine $\mathrm{S}-\mathrm{H}$ group. We validated the sensitivity of this method using bathorhodopsin, a photoproduct of rhodopsin trapped at liquid nitrogen temperature, which undergoes little conformational changes from the dark state as shown by the $X$ ray crystallography. The cysteine residues were individually introduced into 15 positions of Helix III, which contains several key amino acid residues for the lightinduced conformational changes of rhodopsin. The shifts of S-H stretching modes of these cysteine residues and native cysteine residues upon the formation of bathorhodopsin were measured by Fourier transform infrared spectroscopy. While most of cysteine residues demonstrated no shift of $\mathrm{S}-\mathrm{H}$ stretching mode, cysteine residues introduced at positions 117,118 , and 122, which are in the vicinity of the chromophore, demonstrated the significant changes. The current results are consistent with the crystal structure of bathorhodopsin, implying that the cysteine scanning is sensitive enough to detect the tiny conformational changes.

Corresponding author: Yasushi Imamoto, Department of Biophysics, Graduate School of Science, Kyoto University, Kyoto 606-8502, Japan. e-mail: imamoto@rh.biophys.kyoto-u.ac.jp.
Key words: vibrational spectroscopy, conformational change, isomerization, rhodopsin, bathorhodopsin

Protein conformational changes occur in response to stimuli such as ligand, light, voltage, or interaction with other proteins. Because the activity of the functional proteins is closely correlated with their conformation, the activation mechanisms of functional proteins should be understood based on the process of the conformational changes. Because the tertiary structure of the protein is maintained by the intramolecular hydrogen bond, salt bridge, hydrophobic packing etc., perturbation of these intramolecular interactions induces the protein conformational changes. The displacements of atoms upon the conformational changes are detected by X-ray crystallography or NMR. However, the structural analyses of the transient state or the specific conformation in the equilibrium mixture are difficult in general, and the steady methods to probe the intramolecular interaction are desired. Here we propose the "cysteine-scanning" for mapping of the local environmental changes in proteins.

$\mathrm{S}-\mathrm{H}$ group of Cys, whose stretching mode reflects its environment, serves as an excellent vibrational probe ${ }^{1-3}$. Because the S-H stretching mode is well-separated from vibrations of the other groups present in protein, changes in the vibrational mode of Cys can be observed without interference from other vibrational modes, using Fourier transform infrared (FTIR) spectroscopy. In addition, most of cysteine-introduced mutants are functionally expressed by cultured cell lines, because the side chain of Cys is rela- 
tively small and hydrophobic. The tolerance to mutation is essential for the comprehensive analysis.

In the present study, we examined whether or not the environmental changes can be detected by cysteine-scanning using bathorhodopsin (Batho), a photoproduct of rhodopsin trapped at liquid nitrogen temperature ${ }^{4}$. Rhodopsin is a member of G-protein coupled receptors, which have common structural motif composed of seven transmembrane helices ${ }^{5}$. Rhodopsin has an 11-cis-retinal chromophore, which is converted to all-trans form upon photon absorption. Because all-trans-retinal acts as an inherent agonist, photoactivated rhodopsin is eventually converted to active state (metarhodopsin II). However, at liquid nitrogen temperature, the conformation just after photoisomerization is arrested. In fact, the crystal structure of Batho demonstrated that the displacement of atoms are observed only for the chromophore and the adjacent amino acid residues ${ }^{6}$. Therefore, Batho is suitable to validate the sensitivity of cysteine-scanning.

We independently introduced Cys into 15 positions of Helix III because it contains several functionally and structurally important residues, such as Glu113, Glu122, and Glu134 ${ }^{7-11}$. The S-H stretching modes of these Cys residues and native Cys residues were measured by FTIR spectroscopy. While most of Cys residues demonstrated no shift of $\mathrm{S}-\mathrm{H}$ stretching mode, Cys residues introduced in the vicinity of the chromophore (positions 117, 118, and 122) demonstrated the significant changes. The current results are consistent with the crystal structure of Batho, showing the validity of cysteine-scanning.

\section{Materials and Methods}

\section{Preparation of rhodopsin and its cysteine mutants}

Wild-type and mutant bovine opsin genes were expressed in HEK 293S cell lines as previously reported ${ }^{12}$. The pigments were reconstituted by incubating the membrane fragments containing expressed opsins for more than $3 \mathrm{~h}$ at $4^{\circ} \mathrm{C}$ in the presence of 11-cis-retinal. The pigments extracted with $1 \%(\mathrm{w} / \mathrm{v})$ dodecyl- $\beta$-D-maltoside (DM) in buffer $\mathrm{P}$ (50 mM HEPES, $140 \mathrm{mM} \mathrm{NaCl}, \mathrm{pH} 6.5$ ) were incubated with rho 1D4 antibody-agarose gel at room temperature overnight. After washing with buffer A $(0.02 \% \mathrm{DM}$ in buffer $\mathrm{P})$, the pigments were eluted with buffer A containing the $\mathrm{C}$-terminal octadecapeptide of rhodopsin.

Purified rhodopsins were supplemented with a 100 -fold molar excess of L- $\alpha$-phosphatidylcholine (PC) (Sigma type $\mathrm{XI}-\mathrm{E})$ and dialyzed against buffer $\mathrm{P}$ for $3-4$ days at $4{ }^{\circ} \mathrm{C}$ to incorporate pigments into the PC liposomes. Liposomes were then collected by centrifugation, re-suspended in $1 \mathrm{mM}$ phosphate buffer ( $\mathrm{pH}$ 5.7) supplemented with $5 \mathrm{mM} \mathrm{NaCl}$, and placed on $\mathrm{a} \mathrm{BaF}_{2}$ window. The sample dried under vacuum using an aspirator was sealed using another $\mathrm{BaF}_{2}$ window and a spacer after $\sim 1 \mu \mathrm{l}$ of $\mathrm{H}_{2} \mathrm{O}$ was put beside the sample.

\section{Spectroscopy}

The UV-visible absorption spectra of pigments were recorded with a Shimadzu MPS-2000 recording spectrophotometer before the preparation of liposomes. FTIR spectra were recorded using a Bio-Rad FTS40K spectrometer. The temperature of the sample was kept at $78.0 \pm 0.1 \mathrm{~K}$ using an Oxford DN-1704 cryostat and an Oxford ITC-4 temperature controller. Irradiation light was generated using a $1-\mathrm{kW}$ tungsten halogen lamp (Rikagaku Seiki). The Batho minus rhodopsin difference spectra (Batho/Rho spectra) were obtained by alternate irradiations with $501 \mathrm{~nm}$ light passed through an interference filter (Nihonshinku) or $>610 \mathrm{~nm}$ light passed through a glass cutoff filter (R63, Toshiba). For each measurement, 256 interferograms at $2 \mathrm{~cm}^{-1}$ resolution were recorded.

\section{Results}

\section{Effect of the introduction of cysteine residues on} the chromophore vibrational bands in Batho/Rho Spectra

Design of the cysteine-scanning is shown in Figure 1. Among 10 Cys residues in wild-type bovine rhodopsin, Cys322 and Cys323 are palmitoylated, and Cys110 and Cys 187 form a disulfide bond. Thus 6 native Cys residues have free S-H groups. We prepared single mutants for Cys having the free S-H group (C140S, C167S, C185S, C222S, $\mathrm{C} 264 \mathrm{~S}$, and $\mathrm{C} 316 \mathrm{~S}$ ), and double mutant for Cys forming disulfide bond (C110A/187A) to examine the environmental change around these Cys residues in Batho. In addition, environments around Helix III were scanned by 15 cysteineintroduced mutants at positions 117, 118, and 122-134.

All of these mutants were reconstituted into pigments with 11-cis-retinal, indicating that the overall structure of rhodopsin is little perturbed by replacement or substitution of Cys. Figure 2 shows the Batho/Rho spectra of the wildtype and mutants. Absorption maxima of mutants in the dark states are also shown in Figure 2. Whereas the absorption maxima of most mutants were similar to that of wildtype (499 nm), mutations near the chromophore caused relatively large blue-shifts of the absorption maxima (A117C, T118C, E122C and W126C).

Typical chromophore bands of Batho observed in the Batho/Rho spectrum are present at 921,874 and $851 \mathrm{~cm}^{-1}$ in the hydrogen out-of-plane (HOOP) region, 1166 and $1207 \mathrm{~cm}^{-1}$ in the $\mathrm{C}-\mathrm{C}$ stretching region, and $1536 \mathrm{~cm}^{-1}$ in the $\mathrm{C}=\mathrm{C}$ stretching region ${ }^{13}$ (Fig. 2). Even in the mutants exhibiting the blue-shifted absorption maxima, the chromophore vibrational bands in the Batho/Rho spectra were similar to those of the wild-type, indicating that the structure of Batho is not largely perturbed by these mutations. However, the $\mathrm{C}=\mathrm{C}$ stretching bands of $\mathrm{A} 117 \mathrm{C}, \mathrm{T} 118 \mathrm{C}$ and $\mathrm{W} 126 \mathrm{C}$ were slightly up-shifted, reflecting the blue-shifted absorption spectra. The HOOP band at $851 \mathrm{~cm}^{-1}$ and C-C stretching band at $1207 \mathrm{~cm}^{-1}$ of the wild-type were shifted to 861 and $1203 \mathrm{~cm}^{-1}$, respectively, in $\mathrm{T} 118 \mathrm{C}$, suggesting that the amino 


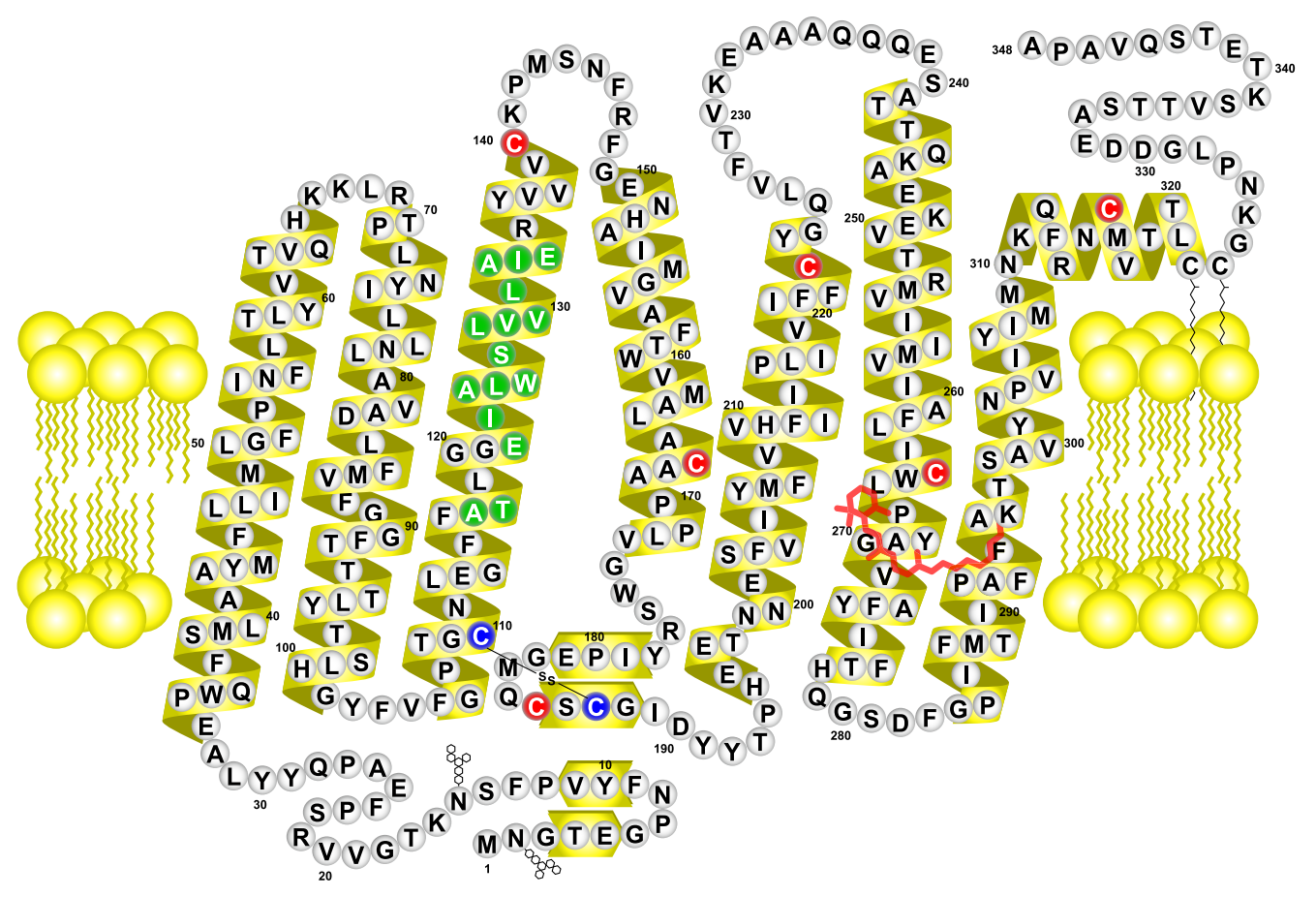

Figure 1 Secondary structural model of bovine rhodopsin. The Cys residues, which have free S-H groups (red), were replaced by serine residues (C140S, C167S, C185S, C222S, C264S, and C316S). Cys110 and Cys187, which form a disulfide bond (blue), were replaced by alanine residues (C110A/C187A). Alternatively, a Cys residue was introduced into Helix III (A117C, T118C, E122C, I123C, A124C, L125C, W126C, S127C, L128C, V129C, V130C, L131C, A132C, I133C, and E134C) (green).

acid residue at position 118 significantly interacts with the chromophore in Batho. The $\mathrm{C}=\mathrm{O}$ stretching bands of the carboxylic acid of Glu122 at 1740, 1735, and $1727 \mathrm{~cm}^{-1} 14$ disappeared in $\mathrm{W} 126 \mathrm{C}$ as well as E122C, probably because the loss of the hydrogen bond between Trp126 and Glu122 perturbs the protonation state of Glu122.

\section{Probing environmental changes by $\mathrm{S}-\mathrm{H}$ stretching mode}

Batho/Rho spectra in the S-H stretching region are shown in Figure 3. A broad positive band was observed at 2610$2540 \mathrm{~cm}^{-1}$, but it was partially beyond the typical S-H stretching frequency $\left(2525-2585 \mathrm{~cm}^{-1}\right)^{1}$. Because Batho/Rho spectra of wild-type rhodopsin expressed in the HEK cells and native rhodopsin isolated from rod outer segments in the S-H stretching region were identical (Fig. 3a), the artifacts arising from using recombinant rhodopsin do not have to be taken into consideration for Batho.

The possible contribution of the $\mathrm{S}-\mathrm{H}$ stretching vibration to the Batho/Rho spectra was examined by using a series of cysteine-replaced mutants (Figs. 3b-3h). Batho/Rho spectra of wild-type (light cyan lines) are superimposed on those of mutants (red lines), and the double difference spectra between wild-type and mutant spectra are shown beneath (blue lines). It is clear that there were no spectral differences between wild-type and cysteine-replaced mutants, indicating that no $\mathrm{S}-\mathrm{H}$ stretching bands of native $\mathrm{Cys}$ residues were involved in the broad positive band of the Batho/Rho spectra.
Cys residues were then introduced into Helix III to probe the environmental changes by observing the S-H stretching vibrations in Batho/Rho spectra (Figs. 3i-3w). Although introduction of Cys residues at most positions did not alter the Batho/Rho spectra in the S-H stretching region (Figs. 31-3w), introduction at positions 117, 118 and 122, which are in the vicinity of the chromophore $(<4 \AA)$, generated additional bands (Figs. 3i-3k). A117C exhibited an intense negative band at $2548 \mathrm{~cm}^{-1}$ and positive band at $2536 \mathrm{~cm}^{-1}$. E122C displayed a moderate negative band at 2575 and positive band at $2570 \mathrm{~cm}^{-1}$. T118C exhibited only a positive band at $2554 \mathrm{~cm}^{-1}$. The bilobic bands in the Batho/Rho spectra of A117C and E122C show the down-shift of the S$\mathrm{H}$ stretching frequencies of Cys117 and Cys122, suggesting that the hydrogen bonds involving S-H groups of Cys 117 and Cys122 were strengthened upon formation of Batho. On the other hand, the presence of only the positive band in $\mathrm{T} 118 \mathrm{C}$ can be explained by either protonation of the thiol anion or a change of intensity without a frequency shift. The former is unlikely because $\mathrm{p} K_{\mathrm{a}}$ of $\mathrm{S}-\mathrm{H}$ is $\sim 9$. Thus, the $\mathrm{S}-\mathrm{H}$ stretching band of Cys 118 would be intensified. It should be noted that no change in S-H stretching mode was observed for W126C, although it shows the significantly blue-shifted absorption spectrum.

Crystallographic analyses have shown the spatial relationship of Ala117, Thr118, Glu122, Trp126, and chromophore in the dark-state and Batho (Fig. 4) ${ }^{6}$. Upon the forma- 

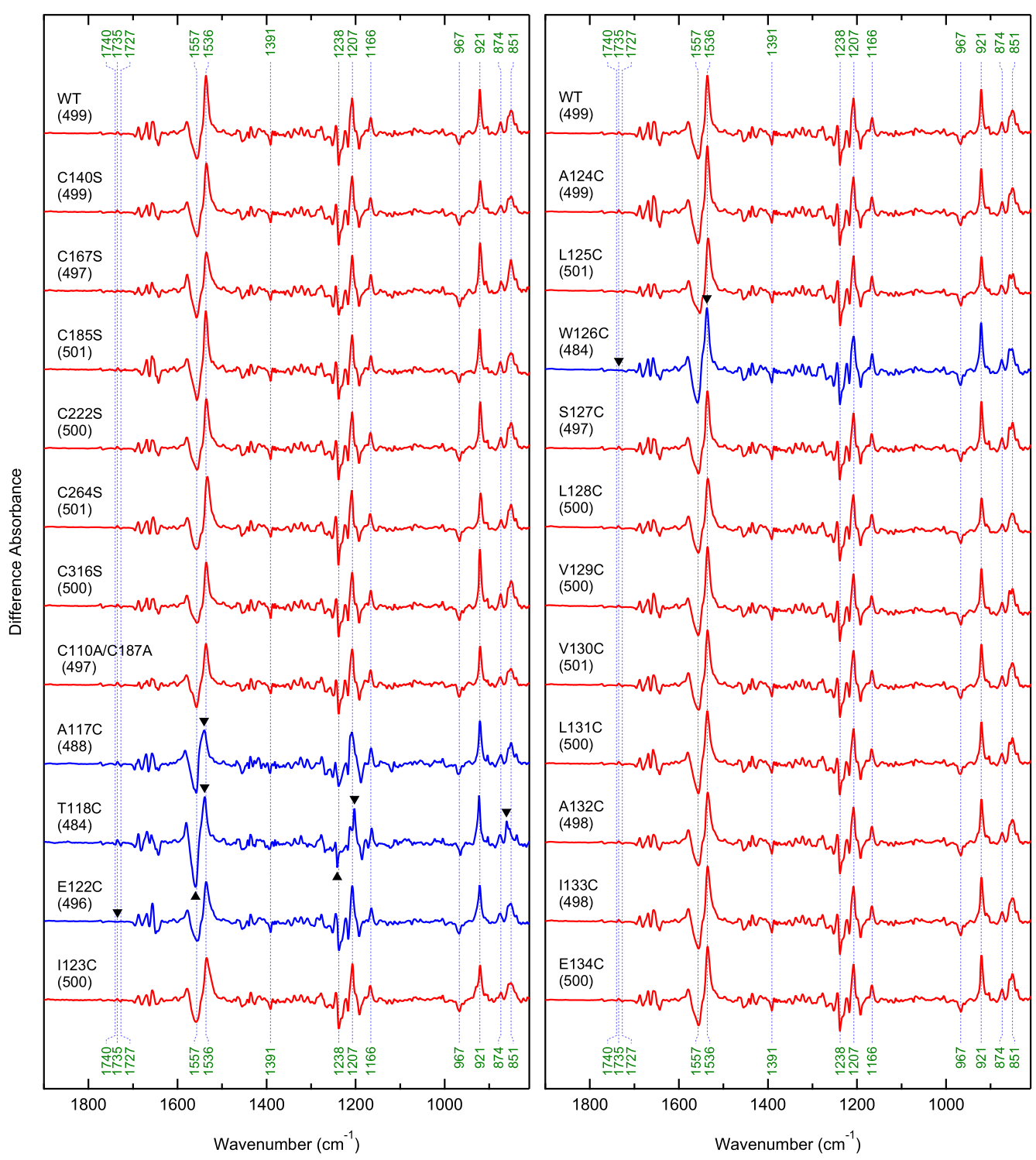

Figure 2 Batho/Rho spectra of wild-type and mutants. Batho/Rho spectra were obtained by irradiation at $78 \mathrm{~K}$. The absorption maxima of the dark states $(\mathrm{nm})$ are shown in the parentheses.

tion of Batho, the distance between Ala117 and the Schiff base linkage of the chromophore is decreased, that between Thr118 and the polyene chain is increased, and that between Glu122 and the $\beta$-ionone ring is decreased (see Discussion). The S-H stretching vibrations of the introduced Cys residues were sensitively altered by these changes in the interactions.

The Batho/Rho spectra of the other mutants showed no significant differences from that of the wild-type in the S-H stretching region, indicating that conformational changes upon formation of Batho are limited in the vicinity of chromophore binding site, as shown by the crystallography ${ }^{6}$.

\section{Discussion}

We successfully detected the local environmental change using cysteine-scanning method, which utilizes the $\mathrm{SH}$ stretching mode of Cys as a sensitive probe ${ }^{1-3}$. The concept of cysteine-scanning is similar to that of recently developed method using a non-natural amino acid $p$-azido-Lphenylalanine $(\mathrm{azF})^{15}$. However, unlike azF, Cys is readily introduced using a well-established point-mutation technique. In addition, due to the smaller size of Cys than azF, introduction of Cys would less perturb the protein conformation than azF.

Broad absorbance increases are observed in the S-H stretching region of Batho/Rho spectra of wild-type. Unexpectedly, the present study clearly showed that they are 


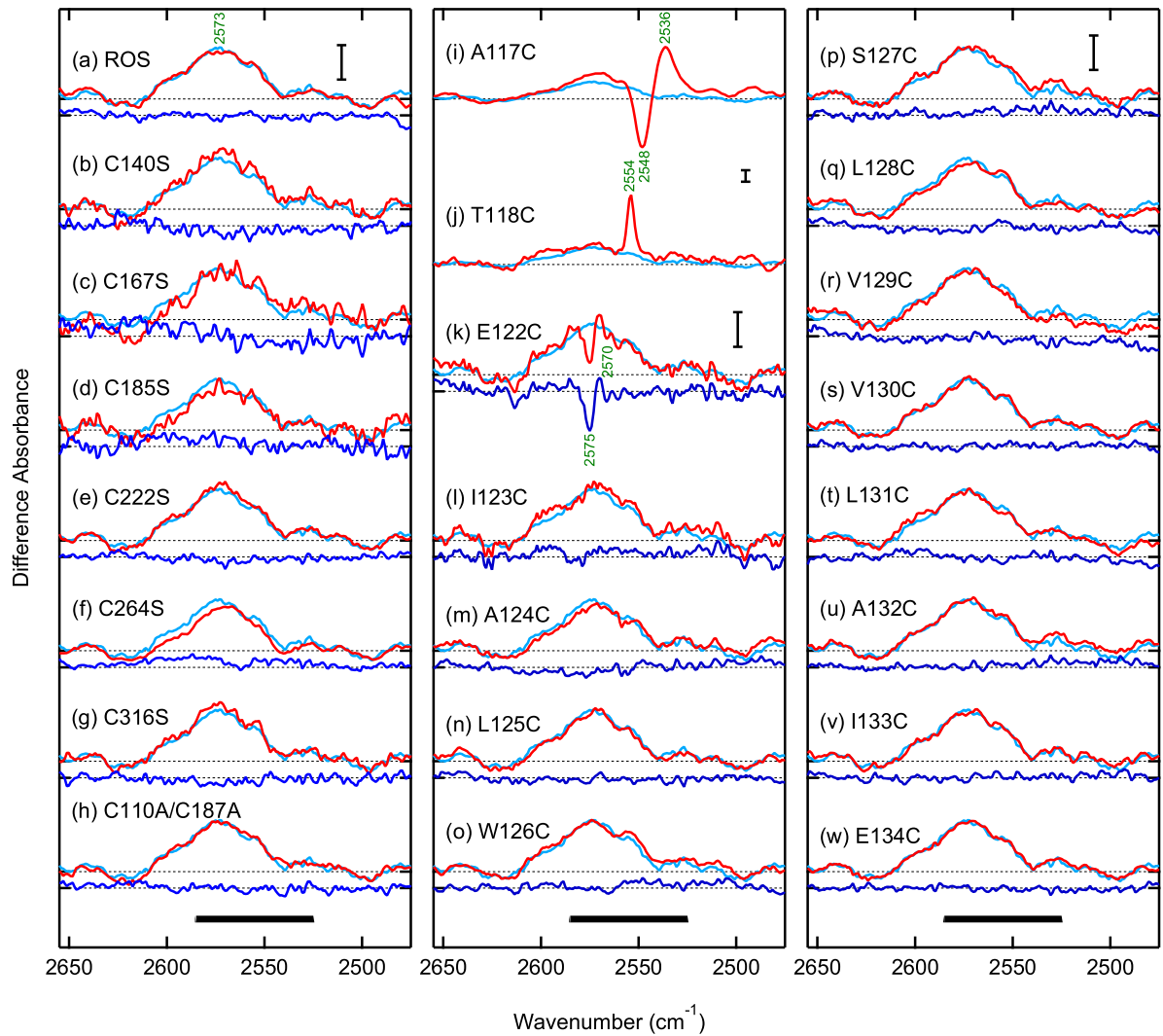

Figure 3 Batho/Rho spectra in the S-H stretching region. Batho/Rho spectra for native rhodopsin in ROS or recombinant rhodopsin mutants (red lines) are superimposed on that of recombinant wild-type (light cyan lines). The double difference spectra are shown beneath (blue lines). Scale bar represents $5 \times 10^{-5}$. Left. Batho/Rho spectra for native rhodopsin in ROS (a) and the cysteine-replaced mutants (b-h). Double difference spectra were calculated by subtracting mutant (or ROS) spectra from wild-type spectra. Middle and right. Batho/Rho spectra for cysteine-introduced mutants $(\mathrm{i}-\mathrm{w})$. Double difference spectra were calculated by subtracting wild-type spectra from mutant spectra. Typical frequency of S-H stretching mode is shown by the thick bar at the bottom of each panel.

attributable to neither the 6 native Cys residues having free $\mathrm{S}-\mathrm{H}$ groups nor the 2 Cys residues forming a disulfide bond. While these bands have not been assigned, it is likely that they are the overtone bands and/or the combination bands of intense chromophore bands of Batho (e.g. the first overtone band of $1207 \mathrm{~cm}^{-1}$ band or combination band of $1536 \mathrm{~cm}^{-1}$ band and $921 \mathrm{~cm}^{-1}$ band).

Among the cysteine-introduced mutants, significant changes of S-H stretching vibration were observed only for A117C, $\mathrm{T} 118 \mathrm{C}$, and E122C upon formation of Batho. The S-H stretching frequency of Cys 117 in A117C shifted from 2548 to $2536 \mathrm{~cm}^{-1}$. Raman spectroscopy of the S-H model compounds showed that $\mathrm{S}-\mathrm{H}$ stretching frequency depends on the strength of the hydrogen bond ${ }^{1}$. The typical frequencies are estimated to be $2585 \mathrm{~cm}^{-1}$ for no hydrogen bond, 2575$2580 \mathrm{~cm}^{-1}$ for a weak hydrogen bond, $2560-2575 \mathrm{~cm}^{-1}$ for a moderate hydrogen bond, and $2525-2560 \mathrm{~cm}^{-1}$ for a strong hydrogen bond. Thus S-H group of Cys117 in the dark state is likely to form a strong hydrogen bond, and it is further strengthened in Batho. Similarly, the hydrogen bond involving Cys122 in E122C is likely to be strengthened in Batho, as shown by the shift from $2575 \mathrm{~cm}^{-1}$ to $2570 \mathrm{~cm}^{-1}$ (Fig. 3k),

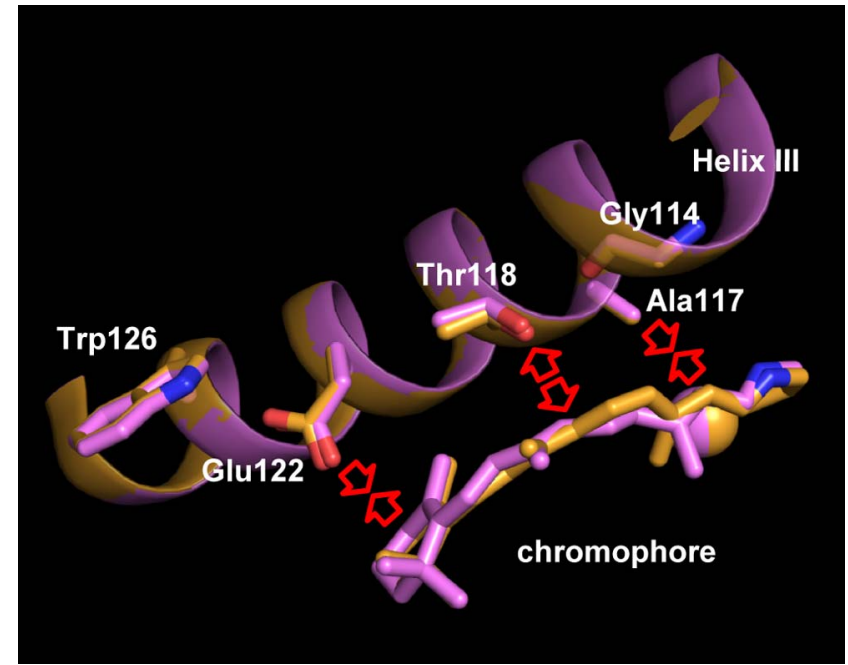

Figure 4 Spatial relationship between Gly114, Ala117, Thr118, Glu122, Typ126, and the chromophore in dark state rhodopsin (orange) and Batho (magenta). The models for dark state and Batho were drawn using $1 \mathrm{U} 19$ and 2G87, respectively. 
while the hydrogen bond of Cys122 is weaker than that of Cys 117. While the hydrogen-bond acceptors of SH groups were not identified in this study, they are likely to be the backbone carbonyl oxygen one helical turn away ${ }^{16,17}$ or the $\pi$-electron system of the chromophore via an SH- $\pi$ hydrogen bond ${ }^{18,19}$.

The crystal structures of the dark state (1U19) and Batho $(2 \mathrm{G} 87)^{6}$ revealed the displacements of the retinal chromophore and its neighboring amino acid residues upon the formation of Batho (Fig. 4). In A-chains of $1 \mathrm{U} 19$ and 2G87, the minimum atomic distance between Ala117 $\left(\mathrm{C}_{\beta}\right)$ and the chromophore $\left(\mathrm{C}_{12} / \mathrm{C}_{14}\right)$ changes from $3.53 \AA$ to $3.40 \AA$, and that between Glu122 $\left(\mathrm{O}_{\varepsilon 2}\right)$ and the chromophore $\left(\mathrm{C}_{5}\right)$ changes from $3.89 \AA$ to $3.49 \AA$. These findings derived from $\mathrm{X}$-ray crystallography are consistent with the vibrational changes of S-H groups at positions 117 and 122, assuming that they interact with the $\pi$-electron system of the chromophore via SH- $\pi$ hydrogen bonds and the strength of the hydrogen bond reflects the donor/acceptor distance.

The S-H stretching band of T118C in Batho/Rho spectrum only appeared on the positive side, suggesting that the intensity of this band was increased with little frequency shift. Unlike SH stretching band of T118C, the OH stretching band of Thr118 in wild-type Batho/Rho spectrum was bilobic. The $\mathrm{OH}$ group of Thr118 forms a hydrogen-bond with backbone carbonyl oxygen of Gly114 both in darkstate (1U19) and Batho (2G87). While the weakening of this hydrogen-bond upon the formation of Batho is suggested by the up-shift of the O-H stretching mode of Thr $118^{20}$, the distance between $\mathrm{O}$ of Gly114 and $\mathrm{O} \gamma$ of Thr118 changes from $2.77 \AA$ to $2.74 \AA$ (or $2.63 \AA$ to $2.56 \AA$ in $\mathrm{B}$ chain). Therefore, the strength of this hydrogen bond does not depend solely on the donor/acceptor distance. The minimum atomic distance between Thr118 $(\mathrm{O} \gamma)$ and the chromophore $\left(\mathrm{C}_{10}\right)$ changes from $3.53 \AA$ to $3.62 \AA$ upon the formation of Batho. Thus alternation of the electrostatic field due to the displacement of the chromophore $\pi$-electron system would weaken this hydrogen bond. Assuming that SH group of Cys 118 is situated similarly to the $\mathrm{OH}$ group of Thr118, electrostatic environment of SH should be altered by the isomerization of the chromophore. Because infrared absorbance is affected by the local polarity ${ }^{21}$, it is likely that the SH stretching band of Cys 118 is intensified by the effect of electrostatic field. However, the lack of the complementary negative band suggests that the effect of electric field on the strength of the hydrogen bond, which correlates with the frequency of the SH stretching band, would be compensated by the shortening of the donor/acceptor distance.

\section{Conclusion}

Cysteine S-H stretching mode is the sensitive probe to detect the local environmental changes. We validated this method using Batho, and demonstrated that the comprehensive introduction of Cys (cysteine-scanning) enables the mapping of the changes in the intramolecular interactions. Cysteine-scanning is applicable for photoactive protein, for which difference FTIR spectra is easily measured. Recently rapid buffer exchange system using attenuated total reflection cell is developed for FTIR ${ }^{22}$. By combination of these techniques, cysteine-scanning is applicable for a variety of proteins.

\section{Acknowledgements}

We are grateful to Prof. Robert S. Molday of University of British Columbia for providing the hybridoma producing rho 1D4 antibody and Dr. Takahiro Yamashita of Kyoto University for providing the rhodopsin E134C mutant plasmid clone. We are also grateful to Dr. Elizabeth Nakajima for critical reading of our manuscript and invaluable comments. This work was supported by Grants-in-Aid for Scientific Research to YY, AT, HK, YS and YI from the Japanese Ministry of Education, Culture, Sports, Science and Technology.

\section{References}

1. Li, H. \& Thomas, G. J., Jr. Cysteine conformation and sulfhydryl interactions in proteins and viruses. 1. Correlation of the Raman S-H band with hydrogen bonding and intramolecular geometry in model compounds. J. Am. Chem. Soc. 113, 456462 (1991).

2. Rath, P., Bovee-Geurts, P. H., DeGrip, W. J. \& Rothschild, K. J. Photoactivation of rhodopsin involves alterations in cysteine side chains: detection of an S-H band in the Meta I $\rightarrow$ Meta II FTIR difference spectrum. Biophys. J. 66, 2085-2091 (1994).

3. Kandori, H., Kinoshita, N., Shichida, Y., Maeda, A., Needleman, R. \& Lanyi, J. K. Cysteine S-H as a hydrogen-bonding probe in proteins. J. Am. Chem. Soc. 120, 5828-5829 (1998).

4. Yoshizawa, T. \& Wald, G. Pre-lumirhodopsin and the bleaching of visual pigments. Nature 197, 1279-1286 (1963).

5. Palczewski, K., Kumasaka, T., Hori, T., Behnke, C. A., Motoshima, H., Fox, B. A., Le Trong, I., Teller, D. C., Okada, T., Stenkamp, R. E., Yamamoto, M. \& Miyano, M. Crystal structure of rhodopsin: A G protein-coupled receptor. Science 289, 739-745 (2000).

6. Nakamichi, H. \& Okada, T. Crystallographic analysis of primary visual photochemistry. Angew. Chem. Int. Ed. Engl. 45, 4270-4273 (2006).

7. Nathans, J. Determinants of visual pigment absorbance: identification of the retinylidene Schiff's base counterion in bovine rhodopsin. Biochemistry 29, 9746-9752 (1990).

8. Fahmy, K. \& Sakmar, T. P. Regulation of the rhodopsintransducin interaction by a highly conserved carboxylic acid group. Biochemistry 32, 7229-7236 (1993).

9. Okada, T., Ernst, O. P., Palczewski, K. \& Hofmann, K. P. Activation of rhodopsin: new insights from structural and biochemical studies. Trends Biochem. Sci. 26, 318-324 (2001).

10. Zhukovsky, E. A. \& Oprian, D. D. Effect of carboxylic acid side chains on the absorption maximum of visual pigments. Science 246, 928-930 (1989).

11. Imai, H., Kefalov, V., Sakurai, K., Chisaka, O., Ueda, Y., Onishi, A., Morizumi, T., Fu, Y., Ichikawa, K., Nakatani, K., Honda, Y., Chen, J., Yau, K. W. \& Shichida, Y. Molecular 
properties of rhodopsin and rod function. J. Biol. Chem. 282, 6677-6684 (2007).

12. Nagata, T., Terakita, A., Kandori, H., Shichida, Y. \& Maeda, A. The hydrogen-bonding network of water molecules and the peptide backbone in the region connecting Asp83, Gly120, and Glu113 in bovine rhodopsin. Biochemistry 37, 17216-17222 (1998)

13. Bagley, K. A., Balogh-Nair, V., Croteau, A. A., Dollinger, G., Ebrey, T. G., Eisenstein, L., Hong, M. K., Nakanishi, K. \& Vittitow, J. Fourier-transform infrared difference spectroscopy of rhodopsin and its photoproducts at low temperature. Biochemistry 24, 6055-6071 (1985).

14. Fahmy, K., Jäger, F., Beck, M., Zvyaga, T. A., Sakmar, T. P. \& Siebert, F. Protonation states of membrane-embedded carboxylic acid groups in rhodopsin and metarhodopsin II: a Fourier-transform infrared spectroscopy study of site-directed mutants. Proc. Natl. Acad. Sci. USA 90, 10206-10210 (1993).

15. Ye, S., Zaitseva, E., Caltabiano, G., Schertler, G. F., Sakmar, T. P., Deupi, X. \& Vogel, R. Tracking G-protein-coupled receptor activation using genetically encoded infrared probes. Nature 464, 1386-1389 (2010).

16. Gregoret, L. M., Rader, S. D., Fletterick, R. J. \& Cohen, F. E.
Hydrogen bonds involving sulfur atoms in proteins. Proteins 9, 99-107 (1991).

17. Rajagopal, S. \& Vishveshwara, S. Short hydrogen bonds in proteins. FEBS J. 272, 1819-1832 (2005).

18. Steiner, T. \& Koellner, G. Hydrogen bonds with $\pi$-acceptors in proteins: frequencies and role in stabilizing local 3D structures. J. Mol. Biol. 305, 535-557 (2001).

19. Meyer, E. A., Castellano, R. K. \& Diederich, F. Interactions with aromatic rings in chemical and biological recognition. Angew. Chem. Int. Ed. Engl. 42, 1210-1250 (2003).

20. Nagata, T., Oura, T., Terakita, A., Kandori, H. \& Shichida, Y. Isomer-specific interaction of the retinal chromophore with threonine-118 in rhodopsin. J. Phys. Chem. A 106, 1969-1975 (2002).

21. Kim, S., Sacksteder, C. A., Bixby, K. A. \& Barry, B. A. A reaction-induced FT-IR study of cyanobacterial photosystem I. Biochemistry 40, 15384-15395 (2001).

22. Furutani, Y., Kimura, T. \& Okamoto, K. Development of a rapid buffer-exchange system for time-resolved ATR-FTIR spectroscopy with the step-scan mode. Biophysics 9, 123-129 (2013). 